\title{
Think national, act local: the role of the physician and the RCP
}

\section{DOI: 10.7861/clinmed.ed.20.2.1}

Decision making in medicine is informed by a subtle mixture of factors: education, experience, local protocols, gut feeling, avoiding litigation. The principle of audit is that one can influence practice also by reflecting back personal performance measured against standardised averages or formal guidance. Mitchell and colleagues highlight some of the challenges herein in their analysis of computed tomography in acute presentations with epilepsy. ${ }^{1}$ They develop the findings of a recent national audit in the UK in selected cohorts of such patients which identified that as many as one in five patients with known epilepsy had scans following presentation to the emergency department. Many of you reading this are involved with such acute medicine presentations. They present a population level analysis over a 3-year period to identify patient factors associated with the rate of scanning. The potential of this to influence management algorithms and practice is discussed elegantly.

The theme of developing local policy by using national datasuch as the Hospital Episode Statistics (HES) - is developed in the paper by Soong et al. ${ }^{2}$ Frailty is a common condition with a potentially significant health impact among older adults. To date, it is not known whether stratifying by degrees of frailty can help plan care and result in improved outcomes. In a retrospective observational study, the authors report the predictive ability of routinely coded 'frailty syndromes' to predict long length of stay and mortality in a 1-year sample of patients over the age of 65 . Frailty is of relevance to generalists, given the previous finding by the same group that the majority of hospital admission patients have at least one of these frailty syndromes.

Clinical Medicine is proudly a journal of the Royal College of Physicians (RCP), and is delighted to showcase topics that are of interest to the RCP. One of these is the need for NHS-based tobacco addiction services, as highlighted in the RCP report Hiding in plain sight: Treating tobacco dependency in the NHS which called for a new model of care providing 'opt-out' specialist services to treat the condition. ${ }^{3}$ Evison and colleagues from Manchester report on the CURE project, the first UK service modelled on the Ottawa Model for Smoking Cessation. ${ }^{4} \mathrm{~A}$ feature of the model is that secondary care clinicians are at the frontline in the delivery of treatment, of especial importance since the NHS 10-year plan commits to funding the treatment of tobacco addiction in hospitals. The CURE project provides a potential framework for national roll out of such services, and we are delighted to present the impact of the study in the first 6 months of service.

Another national, indeed global, health crisis - much more acute - is the ongoing outbreak of the novel coronavirus disease (COVID-19). The RCP, and its journals, are able to respond in nimble fashion with an update on the evolving outbreak, its clinical manifestations and the priorities for the immediate future. Alex Lake, one of the journal's associate editors has provided an excellent overview highlighting principles that physicians should consider. ${ }^{5}$ In this rapidly evolving scenario, the latest advice page remains an essential resource: www.gov.uk/government/ collections/wuhan-novel-coronavirus

As ever, alongside these reviews and original submissions we have our regular CME section (this month, on renal medicine curated by our outstanding demitting nephrology associate editor, Mike Almond) as well as our insight-provoking clinical cases and lessons of the month. And we feature another of our popular Opinion pieces - this time on training in clinical examination. ${ }^{6}$ We hope you find plenty of content to enjoy and share.

\section{References}

1 Mitchell JW, Kallis C, Dixon PA, Grainger R, Marson AG. Computed tomography in patients with epileptic seizures admitted acutely to hospital: A population level analysis of routinely collected healthcare data. Clin Med 2020;20:178-82.

2 Soong JTY, Rolph G, Poots AJ, Bell D. Validating a methodology to measure frailty syndromes at hospital level utilising administrative data. Clin Med 2020;20:183-8.

3 Royal College of Physicians. Hiding in plain sight: Treating tobacco dependency in the NHS. London: RCP, 2018.

4 Evison M, Pearse C, Howle F et al. Feasibility, uptake and impact of a hospital-wide tobacco addiction treatment pathway: Results from the CURE project pilot. Clin Med 2020;20:196-202.

5 Lake MA. What we know so far: COVID-19 current clinical knowledge and research. Clin Med 2020;20:124-7.

6 Dani M, Lee A, Koizia L, Fertleman M. 'Nipples to knees' in the 'Me Too' era. Clin Med 2020;20:235-6.

Anton Emmanuel Editor-in-chief

\begin{tabular}{|c|c|c|c|}
\hline \multicolumn{4}{|c|}{ Members of the editorial board } \\
\hline Michael Almond & Albert Edwards & Yash Mahida & Philip Smith \\
\hline Cono Ariti & Anton Emmanuel & Nick Manning-Cork & Angela Star \\
\hline Paul Belcher & Kate Evans & Chris Marguerie & Cameron Swift \\
\hline Rodger Charlton & Laura Ferrigan & Martin McKee & Rhys Thomas \\
\hline Tim Chevassut & Tevfik Ismail & Andrew Medford & Trainee associate editors: \\
\hline Tahseen Chowdhury & Vikas Kapil & Mehool Patel & Tessa Cacciottolo \\
\hline Richard Corbett & Neelam Kumar & Gerrard Phillips & Rajan Pooni \\
\hline Elaine Dennison & Alexandra Lake & Sushma Saksena & Anenta Ramakrishnan \\
\hline
\end{tabular}

